\title{
Probing Nanoparticle Dynamics in 200 nm Thick Liquid Layers at Millisecond Time Resolution
}

\author{
See Wee Chee ${ }^{1,2}$, Duane Loh $^{1,2}$, Utkur Mirsaidov ${ }^{1,2,3}$ and Paul Matsudaira ${ }^{1}$ \\ 1. Center for BioImaging Sciences, Department of Biological Sciences, National University of \\ Singapore, Singapore 117557 \\ 2. Centre for Advanced 2D Materials and Graphene Research Centre, Department of Physics, National \\ University of Singapore, Singapore 117546 \\ 3. Nanocore, Faculty of Engineering, National University of Singapore, Singapore 117576
}

When imaging specimens in liquids using liquid cell TEM, there is a trade-off between spatial resolution and liquid layer thickness [1]. In TEM mode, the imaging resolution in a liquid cell with 1 micron thick liquid degrades to about $12 \mathrm{~nm}$ for objects near the top $\mathrm{SiN}$ window, due to scattering of electrons by the liquid [1]. While higher resolution can be obtained with thin liquid films (less than $100 \mathrm{~nm}$ thick), the desire for sharper images has to be balanced against keeping the liquid layer thick enough such that processes observed are representative of bulk systems. For example, nanoparticle diffusion observed in thin liquid layers with liquid cell TEM had been found to deviate from the Stokes-Einstein relation [2]. Maintaining a thick liquid layer is also crucial for imaging biological specimens, where, so far, in situ imaging is mainly accomplished using STEM [3]. However, STEM imaging cannot match the temporal resolution of TEM imaging for capturing the motion of dynamic objects where state-of-the-art direct detection cameras are capable of recording whole frames in milliseconds.

Here, we used the motion of $\mathrm{Au}$ nanoparticles as a model system to probe the achievable spatial and temporal resolution in TEM imaging of thicker liquid layers. Au nanoparticles $(\sim 20 \mathrm{~nm}$ in size $)$ are dispersed in water and sandwiched between $30 \mathrm{~nm}$ thick SiN windows in a Hummingbird Scientific liquid flow holder. The holder is loaded into a JEOL 2200FS TEM with an Omega filter, where zero loss imaging (with $20 \mathrm{eV}$ to $40 \mathrm{eV}$ energy slits) was used to mitigate the resolution loss from imaging through the liquid layer (see Figure 1 for a comparison of filtered and unfiltered images). We estimate a typical liquid layer thickness of $\sim 200 \mathrm{~nm}$ at the window edge and $\sim 800 \mathrm{~nm}$ at the window center from electron energy loss spectroscopy (using inelastic mean free paths reported in [4]) for our experiments. Live movies were recorded on a Direct Electron DE-12 camera system at 100 frames per second. Figure 2 shows the trajectory of a single particle near the window edge exhibiting Lévy flight where the motion blur indicates that the nanoparticle made at least two jumps in the $40 \mathrm{~ms}$ time frame. The motion of a pair of connected nanoparticles is described in Figure 3 where it can be seen that during rotation, one of particles moves significantly more than the other. It also shows that our ability to resolve the shape of the nanoparticles is mainly limited by motion blurring.

Particle tracking analysis of the motion of the nanoparticles will be presented. The study of dynamics at millisecond time resolution requires finding an optimal balance between having sufficient signal to noise and minimizing beam induced effects. The nanoparticle dynamics presented here were captured with a dose rate of more than $125 \mathrm{e} /\left(\AA^{2} \cdot \mathrm{s}\right.$ ) (a lower bound calculated from the beam current post-specimen), which can cause significant beam damage (higher dose rates led to dissolution of the nanoparticles). This dose rate will be untenable for imaging soft materials and biological specimens. Strategies for imaging at minimal doses and to retrieve structural information from noisy images will be discussed. 
References:

[1] N. de Jonge and F.M. Ross, Nat. Nanotechnol.6 (2011) p. 695

[2] H.M. Zheng et al., Nano Lett. 9 (2009) p. 2460, J.Y. Lu et al. Nano Lett. 14 (2014) p. 2111.

[3] N. de Jonge et al., PNAS 16 (2009) p. 2159, J.E. Evans et al. Micron 43 (2012) p. 1085, Woehl et al. Sci. Rep. 4 (2014), article 6854.

[4] K.L. Jungjohann et al. Microsc. Microanal. 18 (2012) p. 621.

[5] The authors acknowledge funding from Singapore National Research Foundation's Competitive research program funding (NRF-CRP9-2011-04) and thank Ms. S.F. Tan and Dr. C.A. Nijhuis for their assistance in providing the nanoparticles.

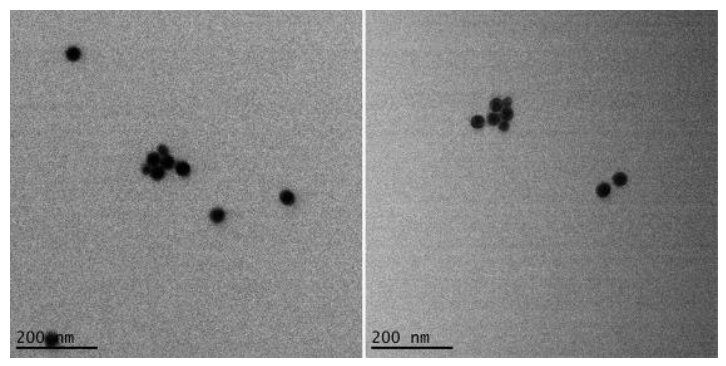

Figure 1. Bright field TEM images of Au nanoparticles in water imaged (left) without energy filtering and (right) with a $20 \mathrm{eV}$ energy filter inserted

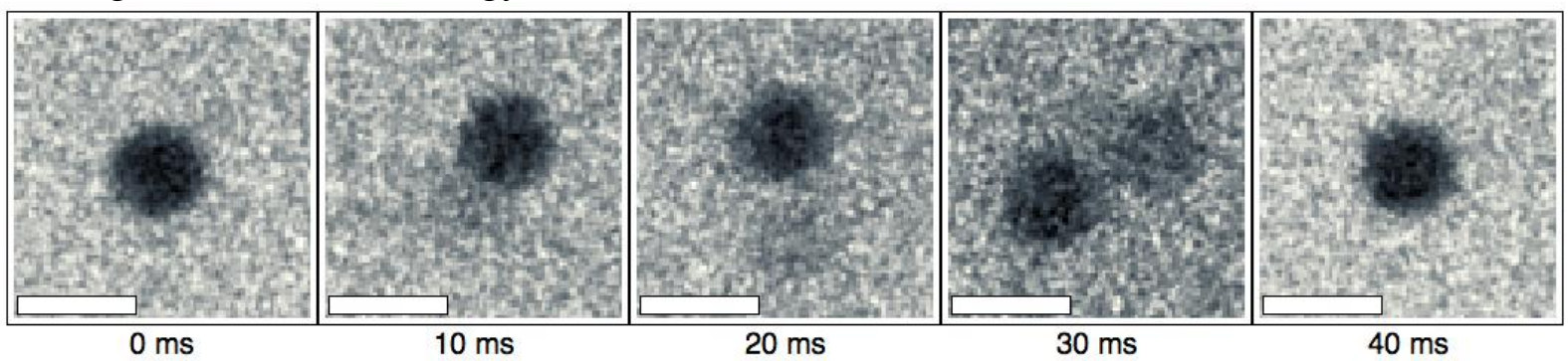

Figure 2. $40 \mathrm{~ms}$ sequence of a nanoparticle near the window edge in Lévy flight ( $40 \mathrm{eV}$ energy slit). The motion blur indicates that the nanoparticle made at least two jumps. Scale bar represents $20 \mathrm{~nm}$.

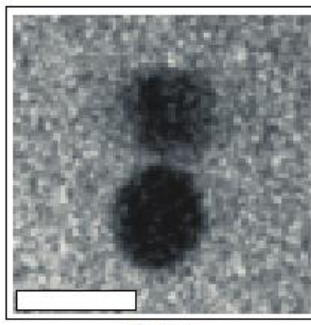

$0 \mathrm{~ms}$

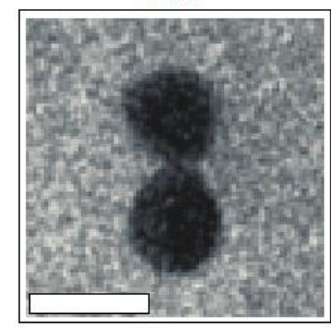

$50 \mathrm{~ms}$

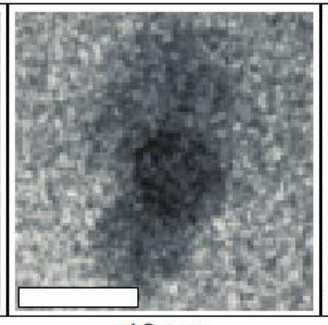

$10 \mathrm{~ms}$

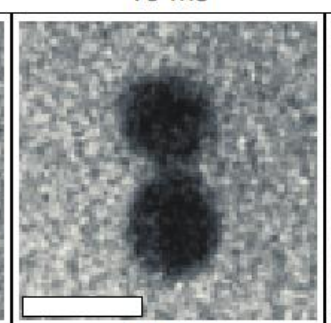

$60 \mathrm{~ms}$

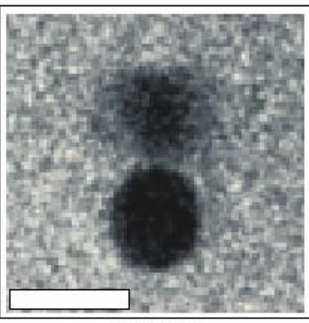

$20 \mathrm{~ms}$

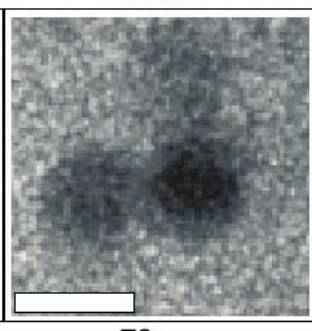

$70 \mathrm{~ms}$

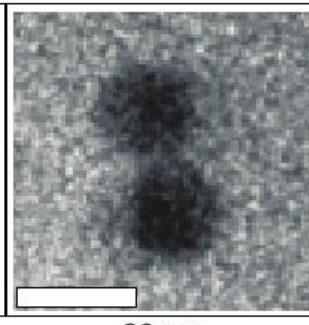

$30 \mathrm{~ms}$

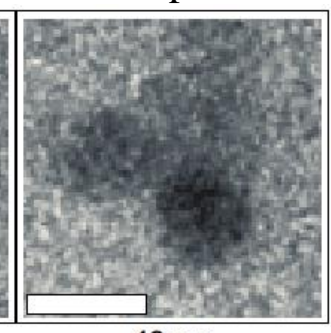

$40 \mathrm{~ms}$

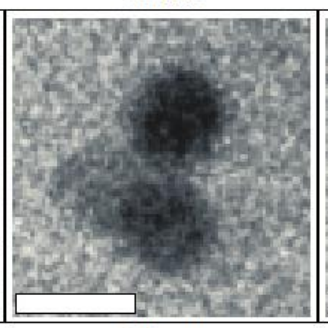

$80 \mathrm{~ms}$

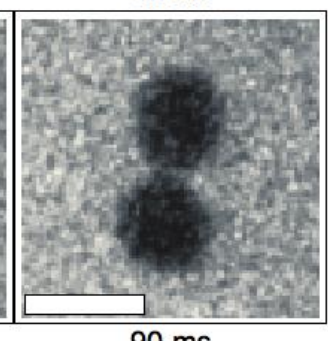

Figure 3. $90 \mathrm{~ms}$ sequence of a two nanoparticle pair rotating ( $20 \mathrm{eV}$ energy slit). The images show that one nanoparticles moves significantly more than the other. Scale bar represents $20 \mathrm{~nm}$. 